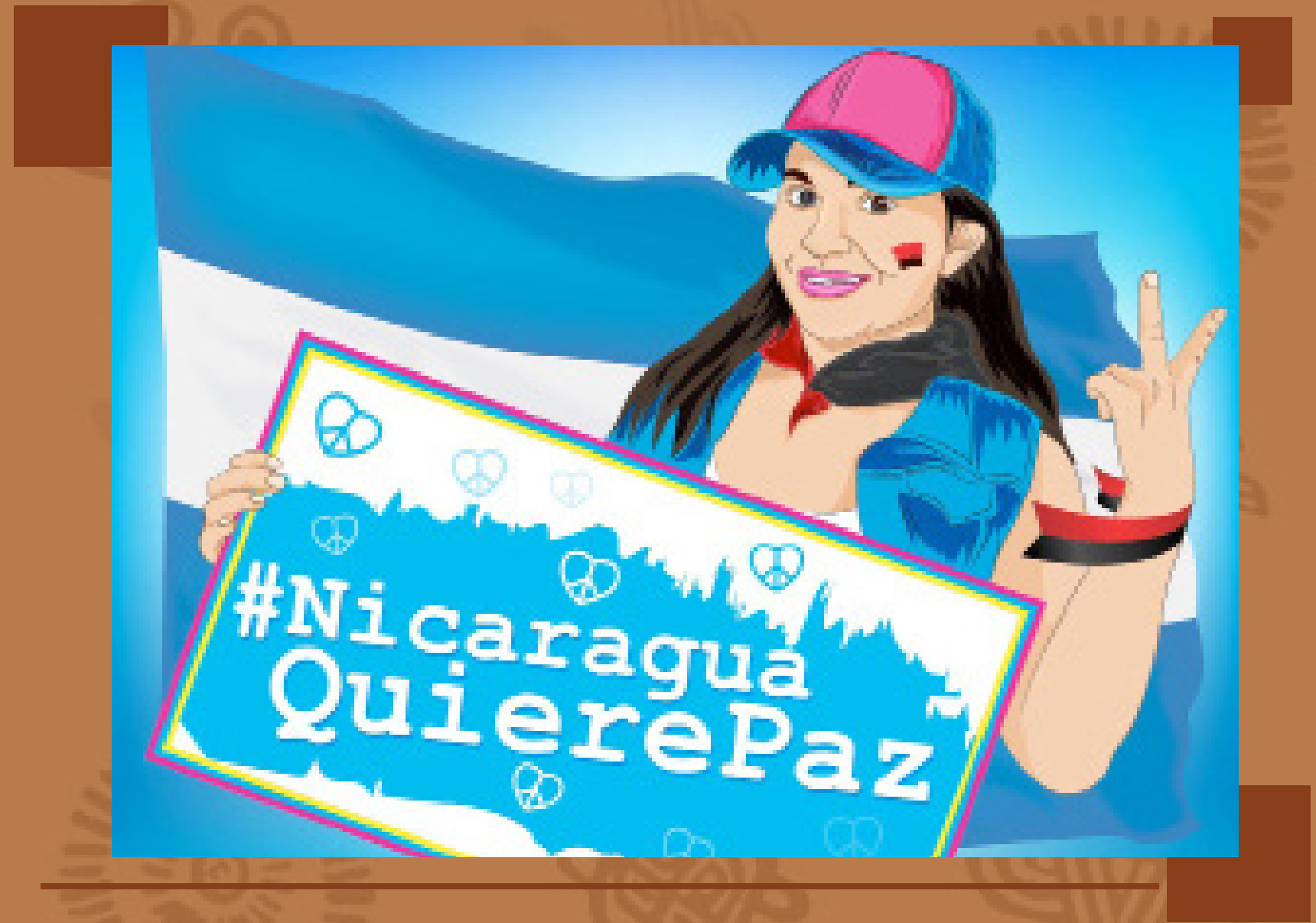

\title{
Implementación del programa cultura de paz y el rol del docente en la UNAN Managua
}




\section{Implementación del programa Cultura de Paz y el rol del docente en la UNAN Managua.}

\section{Implementation of the Culture of Peace program and the role of the teacher at UNAN Managua.}

\section{Danny Valeria Benítez Mayorga}

Docente del departamento de Filosofía, de la carrera Ciencia Política y Relaciones

Internacionales, UNAN-Managua

https://orcid.org/0000-0002-7838-1638

danny.benitez@unan.edu.ni
Recibido: 20-04-2021

Aceptado: 28-05-2021

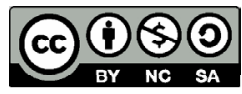

Copyright $\odot 2021$ UNAN-Managua Todos los Derechos Reservados.

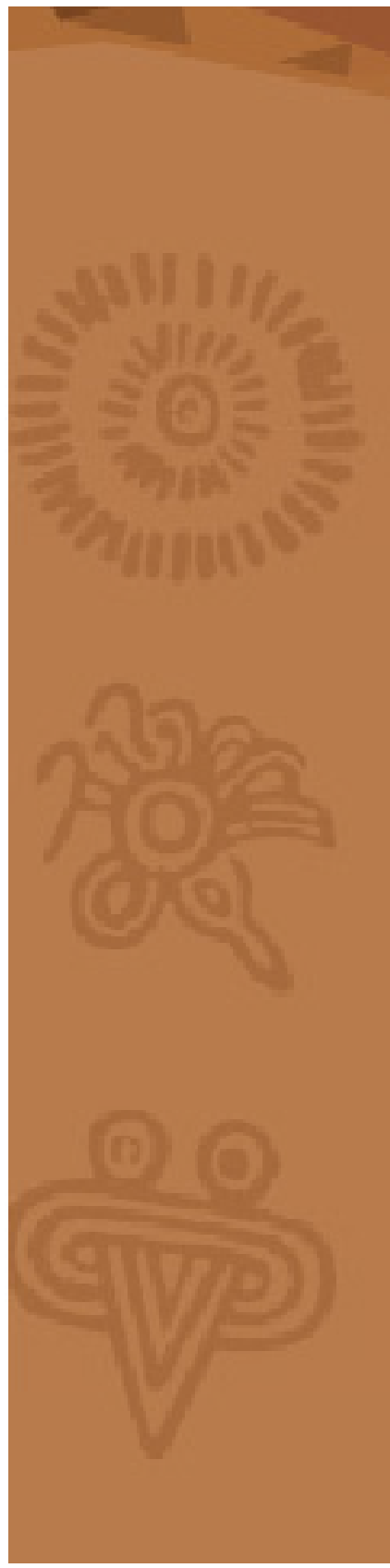

\section{Introducción}

El presente ensayo tiene como finalidad dar a conocer la importancia de la implementación del programa Cultura de Paz y el rol del docente en la UNAN Managua, según la definición de las Naciones Unidas en la resolución del año 1999, establece que; "la cultura de paz consiste en una serie de valores, actitudes y comportamientos que rechazan la violencia y previenen los conflictos tratando de atacar sus causas para solucionar los problemas mediante el diálogo y la negociación entre las personas, los grupos y las naciones". (Unesco, 2000)

El estudio de, cómo se asume el programa cultura de paz en las universidades públicas, principalmente en la UNAN Managua es parte vital de las acciones que se desempeñan en la docencia, en el quehacer de cada miembro de la comunidad, donde trabajadores y estudiantes deben convivir de manera armoniosa en irrestricto respeto a los derechos humanos, en pleno reconocimiento del yo y del otro, ambos como sujetos de los mismos derechos.

En el trabajo se realiza una descripción del rol del docente universitario y la importancia del mismo, fundamentado en opiniones de expertos en el tema y desde la perspectiva como docente de esta Alma Mater, para promover y propiciar los planteamientos establecidos en el programa "Cultura de Paz", que se implementa desde el año 2019 en la institución.

Parafraseando al Diputado Carlos Emilio López, quien es el coordinador nacional de la comisión de Reconciliación, Justicia y Paz; El programa cultura de paz es, "La aplicación y desarrollo de una serie de temáticas, con enfoque de género, igualdad, equidad, justicia social, convivencia pacífica y sana, unidad y colaboración entre los habitantes de un territorio", es por ello que, en este análisis, se indagará los beneficios que conlleva la ejecución del programa en la comunidad universitaria.

Para realizar este trabajo, se efectuó un análisis documental desde los antecedentes y punto de partida de la Cultura de Paz para ayudar a los pueblos a emerger de los conflictos, se tuvo acceso a distintas entrevistas, entre ellas, las del Diputado Carlos Emilio López, la Rectora de la UNAN Managua y presidenta del CNU, maestra Ramona Rodríguez, así como a la Rectora de la UNAN León, maestra Flor de María Valle y por supuesto las distintas intervenciones de la Vicepresidenta compañera Rosario Murillo quien de manera reiterada hace énfasis en la importancia de la paz, la armonía, solidaridad y convivencia armoniosa entre los hermanos nicaragüenses, así mismo se llevó a cabo la revisión de diversas fuentes y estudios del impacto del programa, además del sondeo y consulta a docentes y estudiantes que han estado inmersos en todo el proceso. 
(Santibáñez, 2011) La Cultura de Paz se convierte en un componente fundamental del ciudadano, que, como persona con derechos y responsabilidades, construye ciudadanía y asume un rol de agente transformador en una sociedad, que, bajo los valores de la democracia y la libertad, busca el desarrollo y el bienestar social de todos por iguales (pagina 9).

Siendo entonces la cultura de paz un elemento fundamental en todos los procesos de la vida del ser humano, por medio de la cual pueden emerger los cambios sociales y las transformaciones dentro de la sociedad, las que vendrían a impactar de manera positiva todo el entorno en el cual se llevan a cabo los procesos de desarrollo que permiten el crecimiento integral de los seres humanos y los colectivos sociales.

\section{Desarrollo}

El diputado Carlos Emilio López afirma que la cultura de paz "es la cultura de la igualdad entre hombres y mujeres", de igual manera expresa que, "en la Constitución Política de Nicaragua, se reconoce los derechos de hombres y mujeres", y que estos derechos deben de respetarse sin discriminaciones, "y también dice la ley superior que el Estado tiene la obligación de remover todos los obstáculos que impidan el ejercicio de igualdad entre hombres y mujeres". (CNU, 2019).

Lo que significa que el Estado por medio de la legislación y las instituciones públicas, debe ser garante de que estos derechos sean cumplidos y respetados por todos los ciudadanos, para ello debe dotar a la sociedad por medio de diversas acciones, de los elementos necesarios para ese cumplimiento, es por tal motivo que surge la necesidad de concretar acciones enfocadas en el área de derechos humanos, y convivencia pacífica entre los pueblos.

El programa «Universidad para la Paz» promueve una cultura de armonía nacional. En la apertura del programa el día lunes 15 de julio de 2019 en la UNAN-León, destacaron las palabras de la Maestra Ramona Rodríguez, rectora de la UNAN Managua quien señala;

"La construcción de una cultura de paz en Nicaragua ha sido un derecho y es un derecho irrenunciable, por eso el CNU acordó la creación del programa Universidad para la Paz porque la educación desempeña un rol fundamental en la formación de valores y en el desarrollo de la convivencia lo cual es imprescindible para la coexistencia pacífica, armoniosa y de respeto mutuo entre las personas". (La Voz del Sandinismo, 2019)

Por consiguiente, se identifica la trascendencia del programa para la comunidad universitaria, jóvenes, adultos, docentes y personal administrativo, están presentes e inmersos en el mismo, la paz es un proceso que todas y todos debemos construir, por medio de la educación, la sana convivencia y adoptando cada uno las acciones necesarias para la coexistencia pacífica y armoniosa, la paz es un trabajo de todos, pero sobre todo debe emerger de las universidades y reflejarse en la sociedad. 


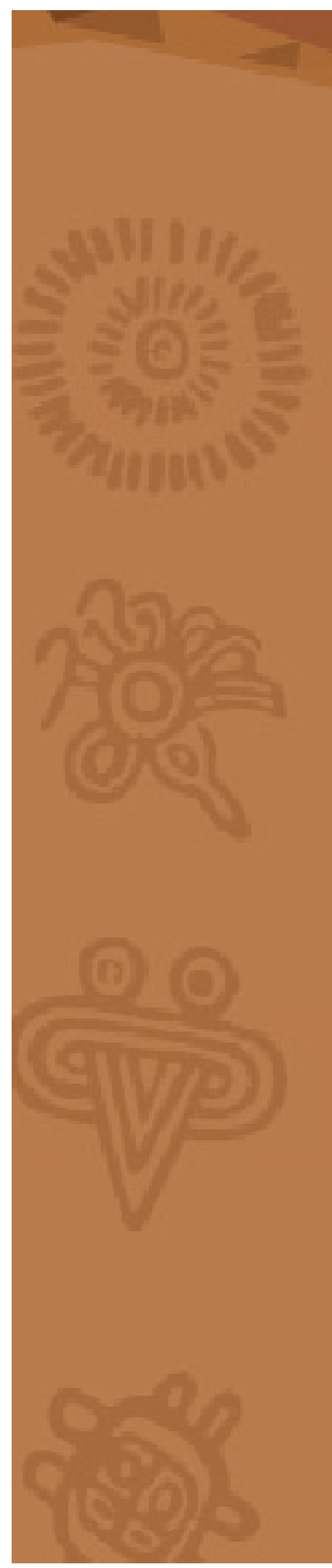

El programa Universidad para la Paz se basa en tres pilares:

Uno relacionado con la Cultura de la Paz tomando en cuenta la igualdad de género; la relación intergeneracional, el amor a la Madre Tierra y la Interculturalidad, un segundo pilar vinculado a los derechos constitucionales y restitución de derechos que promueve el gobierno sandinista con todos y cada uno de los programas sociales y socio productivos. El tercer pilar radica en la importancia que tiene vivir en paz para fomentar el crecimiento y el desarrollo de la nación. (Ortega, 2019).

Por lo tanto, se debe comprender la cultura de paz desde un enfoque multidimensional, ya que esos tres pilares abordan todo el quehacer y sentir de todos los sectores de la sociedad, se integra de manera armoniosa a todos los ciudadanos, a quienes se les garantiza y restituyen derechos, se trata de fomentar y aprovechar las distintas capacidades de todas y todos, sin distingos ni discriminaciones de ninguna índole.

Asimismo, la rectora de la Universidad Nacional Autónoma de León, UNAN León, la maestra Flor de María Valle, hace referencia a la importancia del Programa y Lanzamiento de Cultura de Paz, por medio de "Universidad para la Paz", al expresar que, "Es nuestra obligación trabajar en la promoción de una cultura de paz que nos conlleve a practicar valores y actitudes de respeto, solidaridad y amor a la vida, aspectos fundamentales para lograr la estabilidad y desarrollo humano sostenible que anhelan las familias nicaragüenses". (Ortega, 2019).

Al señalar que es nuestra obligación trabajar en la promoción de una cultura de paz, la rectora hace clara referencia al trabajo que se ejerce desde las universidades en pro del servicio de la sociedad, y principalmente desde la docencia, donde los maestros trabajan en aras de la formación de estudiantes integrales, con sentido humanista, compromiso social y ético.

Lo puntualizado por los catedráticos compromete, aún más, el trabajo de los docentes dentro de la comunidad universitaria, ya que para alcanzar la equidad de género, lograr la interculturalidad de los pueblos, fomentar el desarrollo, la convivencia sana y pacífi$\mathrm{ca}$, armoniosa, en igualdad de condiciones y lograr el crecimiento del país, se necesita de docentes comprometidos con el cambio social, que practiquen y fomenten valores, por medio de una cultura de dialogo, solidaridad y amor, que son los elementos que anhelan las familias nicaragüenses para alcanzar una vida plena.

Esto conlleva firmemente a la puesta en práctica de la reconciliación entre los miembros de una colectividad, comunidad, sociedad o familia, al pasar por un conflicto, crisis o desacuerdos que generen confrontaciones, es esencial identificar las causas de las desavenencias y proponer alternativas para solucionar tales situaciones.

Para (Torres, 2019) La Reconciliación comprende el reconocimiento de nuestras propias faltas, la Reconciliación es pactar la paz con la tierra como elemento y Casa de la Humanidad. La Reconciliación es una aspiración y práctica de la humanidad para garantizar estabilidad y paz duradera (pag.4). 
Dicha conceptualización reafirma el hecho de que, para la superación de un conflicto, debe existir antes una reflexión interna, una asimilación de las situaciones a las que se enfrenta, la construcción de cada uno de manera individual para una posterior construcción de una colectividad con bases comunes en la búsqueda de soluciones que garanticen la paz real, eficaz y prolongada.

\section{El rol docente en el programa de Cultura de Paz}

Se puede afirmar que en la UNAN Managua se promueve y fomenta la Cultura de Paz por medio de la educación inclusiva y de calidad, a través de las distintas actividades de extensión universitaria, una extensión que asume los diversos modelos y procesos extensionista, pasando por medio de una educación critica, transformadora y liberadora, donde los miembros de la comunidad universitaria se sumergen en la identificación de las problemáticas sociales que frenan el desarrollo y afectan a los miembros de la sociedad, en la búsqueda constante de soluciones a los mismos.

De igual manera por medio de la vinculación de la Universidad-Sociedad-Estado, se fortalece el rol del docente dentro de la comunidad universitaria, a través de la atención a los becados internos, con en el trabajo del movimiento estudiantil, donde se da especial énfasis en los programas del sistema educativo superior público, se fomenta la paz, los derechos humanos, la tolerancia, el entendimiento ante la diversidad y la interculturalidad, la democracia, la no violencia y el respeto.

El rol de los docentes es fundamental en las aulas de clases, en la comunidad universitaria en general, y en la sociedad en su conjunto, los docentes representan para la universidad los garantes del acompañamiento en el proceso de formación de profesionales integrales, con sentido humanista, ético, con compromiso social, e identidad institucional, asimismo, el fomento de una Cultura de Paz es sustancial para lograr la armonía y la buena andanza entre cada uno de los miembros de la comunidad universitaria.

\section{El diputado Carlos Emilio, lo expresa de la siguiente manera;}

Que los estudiantes respeten a los docentes, que los docentes respeten a los estudiantes; que respetemos a los adultos mayores, que respetemos a los pueblos originarios y afrodescendientes, que todas las culturas, reconociendo, como dice la Constitución Política, que somos una Nación, multicultural, plurilingüe y multiétnica, respetemos esa diversidad cultural (El 19 Digital, 2018)

La sociedad nicaragüense está inmersa en la tarea de construir una Cultura de Paz y una práctica de valores que trascienda las aparentes diferencias que se pueden tener como sociedad, y unirse en la búsqueda de objetivos colectivos y metas comunes, la cual llevará a los integrantes de la comunidad a alcanzar mejores condiciones, en todos los aspectos; sociales, políticos, culturales, entre otros. 
El papel protagónico que tiene cada docente radica en la capacidad de transmitir valores y relacionarse en el entorno, por medio de las distintas funciones y actividades dentro y fuera de la comunidad universitaria, para generar y promover prácticas de Cultura de Paz y Buen Vivir entre todos sus miembros, debe entenderse como la capacidad que este tiene de no solo buscar concluir un semestre de clase para subir notas a un sistema, y así cumplir con un acta que determine cuántos estudiantes aprobaron o no, sino que el papel del docente en la universidad hoy en día, trasciende esta incluye la capacidad que tuvo ese docente para integrarse en distintas actividades de la comunidad universitaria, ya que solo se podrá fomentar una Cultura de Paz cuando cada docente se sienta parte inalienable de la institución.

La crisis sociopolítica que atravesó el país en el 2018, evidenció que la paz es un derecho por el cual se debe luchar, por medio de la docencia se va a dota a los estudiantes y a todos los miembros de la comunidad universitaria de las herramientas, para que pueden identificar cuando un conflicto trasciende, al punto de dividir a la sociedad, a las familias, las relaciones interpersonales y se les obliga de manera consciente o inconsciente a tomar bandos o posturas, a causa de la intolerancia y la falta de respeto ante la diversidad de pensamientos e ideologías.

Es ahí donde queda aún más en evidencia, lo importante que es para la sociedad nicaragüense, para los jóvenes, el trabajo de los docentes comprometidos con su profesión y con los estudiantes, que buscan en ellos una guía para desaprender las actitudes que frenen su desarrollo y crecimiento personal y profesional.

Puede concluirse que, las universidades miembros del CNU están siendo consecuentes con lo que plantea la Organización de las Naciones Unidas y lo que demanda la sociedad nicaragüense y el Estado garantiza, para una educación en el contexto actual, que es educación para la paz, en pro de la defensa de los derechos humanos, para la construcción de justicia y la eliminación de las desigualdades y la violencia. Así mismo este programa cultura de paz, el que se cumple en la UNAN Managua, por medio de "Universidad para la Paz" se vincula también, con los distintos objetivos de desarrollo sostenible, donde se plantea el tema de la paz, justicia, e instituciones fuertes, para eliminar la violencia.

En un escenario posterior a un conflicto, es necesario se trabaje en la búsqueda de soluciones, reconciliación y superación de las controversias, hablar de paz es hablar de comunidad, retomar proyectos y programas que incluyan a todos los miembros de la sociedad, sin distingos, ni credos políticos o religiosos.

El estudio de la cultura de paz, es imprescindible para los miembros de la comunidad universitaria, y sobre todo para los docentes universitarios quienes tienen rol y tarea de reflejar esos pilares que se plantean en el programa, en el desarrollo de sus actividades, por medio de su comportamiento y con las relaciones interpersonales que establecen dentro de la comunidad universitaria. La creación de este programa es de vital relevancia porque la educación, desempeña un rol fundamental en la formación de valores y en el desarrollo de la convivencia, pacifica, sana, y armoniosa, para lo cual es necesario el respeto mutuo entre las personas, es el camino para vivir en solidaridad y unidad los unos con los otros. 
Forjar una cultura de paz en la comunidad universitaria es hacer que las autoridades, docentes, trabajadores administrativos y estudiantes, comprendan y respeten la libertad, la justicia, la democracia, los derechos humanos, la tolerancia, el respeto, la igualdad y la solidaridad. Todo en conjunto apunta a un rechazo colectivo a la violencia, la suma en la promoción de la participación activa en el quehacer de toda la comunidad universitaria, la familia, los hombres, mujeres y niños, en el centro de la sociedad.

El respeto, la tolerancia, los derechos humanos, la justicia y democracia, preservación de la cultura, resolución pacífica de conflictos, entre otros, son temas vitales a la hora de hablar de paz, estos temas deben ser desarrollados por los docentes en las aulas de clases y en todos los entornos sociales, hablar de paz es hablar de diversidad, inclusión, en cuanto a la educación, es hablar de calidad y pertinencia, esos elementos se han reflejado tanto en el programa "Universidad para la Paz" como en el rol que los docentes desempeñan en la UNAN Managua.

\section{Referencias}

CNU. (16 de Julio de 2019). CNU. Obtenido de http://www.cnu.edu.ni/cultura-de-pazen-las-universidades-miembros-del-cnu/

El 19 Digital . (13 de Noviembre de 2018). El 19 Digital . Obtenido de https://www. el19digital.com/articulos/ver/titulo:83869-diputado-carlos-emilio-lopez-explica-alcances-de-politica-de-estado-para-una-cultura-de-reconciliacion-y-paz

La Voz del Sandinismo. (15 de julio de 2019). Programa «Universidad para la Paz» promueve una cultura de armonía nacional. Recuperado el 11 de Marzo de 2020, de La Voz del Sandinismo: https://www.lavozdelsandinismo.com/nicaragua/2019-07-15/ programa-universidad-para-la-paz-promueve-una-cultura-de-armonia-nacional/

López, C. E. (15 de Julio de 2019). Premisas Fundamentales para una Cultura de Paz. Lanzamiento de Programa Cultura de Paz.

Ortega, P. (15 de Julio de 2019). El 19 Digital. Obtenido de https://www.el19digital.com/articulos/ver/titulo:92128-realizan-en-leon-lanzamiento-oficial-del-programa-universidad-para-la-paz

Santibáñez, G. G. (2011). Cultura de Paz y reforma democrática de la institucionalidad en Nicaragua. Managua, Nicaragua: Centro de Investigación y de Estudios Latinoamericanos y Caribeños, UPOLI Nicaragua. Obtenido de http://biblioteca.clacso.edu.ar/Nicaragua/cielac-upoli/20170821080800/Libro-Cultura-de-Paz-y-Reforma-Democratica. pdf

Torres, D. (Enero-Abril de 2019). Hacia una Cultura de la Educación . Cultura de Paz, 24(74), 1-4. Obtenido de http://revistasnicaragua.net.ni/index.php/culturadepaz/article/ view/5753/6612 
Danny Valeria Benítez Mayorga

Docente del departamento de Filosofía, de la carrera Ciencia Política y Relaciones Internacionales, UNAN-Managua (2014-2021). Maestrante del programa Sociología del Desarrollo, Licenciada en Diplomacia y Ciencias Políticas, Delegada de la Función Extensión del departamento, Jurado en Jornadas de Desarrollo Científico JUDC, Facultad de Humanidades y Ciencias Jurídicas. Ponente en IV Congreso Nacional de la Educación Superior Nicaragüense. Con diplomado en "Juventudes: Historia, Realidades y Desafíos (Instituto Nicaragüense de juventudes (INJUVE y UNAN Managua). Actualmente estudiante de diplomado en Extensión Universitaria. Seminarios en "Derecho Comunitario en Centroamérica (Asociación de Estudiantes de Derecho de la UNAN Managua y la Federación de estudiantes de Derecho de las Universidades Estatales de Centroamérica). Seminario-Taller sobre Derechos del Consumidor. Y en marco de políticas públicas medioambientales. Con cursos en Innovación y Emprendimiento "Como crear tu startup desde el enfoque de emprendimiento", Actualización en Redacción científica, Edición y visibilidad de revistas científicas. Taller de Periodismo Científico “Unidos en la popularización de la Ciencia", CONICYT. Obtuvo el Diploma de reconocimiento por labor como dirigente estudiantil, y particularmente como miembro del consejo de la Facultad de Humanidades y Ciencias Jurídicas en el año 2013. 